\title{
Up-regulation of A20/ABIN1 contributes to inefficient M1 macrophage polarization during Hepatitis $C$ virus infection
}

\author{
Chao Fan ${ }^{\dagger}$, Ying Zhang ${ }^{\dagger}$, Yun Zhou, Bingjie Li, Yu He, Yonghong Guo and Zhansheng Jia
}

\begin{abstract}
Background: Anti-hepatitis C virus (HCV) responses are often accompanied by an increase in alanine aminotransferase levels in HCV-infected patients, indicating that inflammatory responses are compromised by the virus. Additionally, inflammation is associated with M1-polarizated macrophages, which secrete cytokines such as tumor necrosis factor-a, interleukin-1, and interleukin-12, and present antigens through phagocytosis. HCV-encoded proteins are presented as specific viral antigens in particular infectious steps that influence the immune response. For instance, HCV antigens impact macrophage PD-1 and Tim-3 expression, and contribute to impaired viral clearance. Furthermore, circulatory HCV antigens from infected patients inhibit dendritic cell differentiation, which raises the possibility that HCV antigens may also interfere with macrophage polarization.
\end{abstract}

Methods: In this study, the impact of HCV antigen stimulation on M1-polarized macrophages was investigated. The influence of HCV antigens was evaluated by reverse transcription polymerase chain reaction and enzyme-linked immunosorbent assay. Specific changes were investigated clinically by flow cytometry and immunofluorescence. Effects of NF-KB during the process were analyzed by western blot.

Results: HCV infection dampened M1 macrophage polarization ex vivo and in vitro. After antigen stimulation, NF-KB signaling was suppressed by the up-regulation of A20 and A20-binding inhibitor of NF-KB binding protein, which likely leads to a variation of functional molecules such as tumor necrosis factor-a, CD163, matrix metalloproteinases, transferrin receptor-1, and CD100, reflecting an anti-inflammatory reaction against M1-polarization.

Conclusion: HCV antigens stimulation up-regulates A20/A20-binding inhibitor of NF-KB binding protein expression, which consequently contributes to inefficient M1 macrophage polarization.

\section{Background}

Hepatitis $\mathrm{C}$ virus $(\mathrm{HCV})$ accounts for millions of viral hepatitis cases each year [1]. Because most hepatitis symptoms are mild or even absent, patients often fail to report chronic infection later in life. Anti-HCV responses are often accompanied by an increase in alanine aminotransferase (ALT) levels. Patients exhibit persistent mild up-regulated ALT in chronic HCV, indicating that the virus may dampen inflammatory responses. Inflammation is associated with professional antigen presenting cells (APCs) including dendritic cells (DCs) and macrophages that secrete cytokines such as tumor necrosis

\footnotetext{
* Correspondence: jiazsh@fmmu.edu.cn

${ }^{\dagger}$ Equal contributors

Department of Infectious Diseases and Center of liver Diseases, Tangdu Hospital, the Fourth Military Medical University, Xi'an 710038, China
}

(TNF)- $\alpha$ and interleukin (IL)-12, and present antigens for $T$ and $B$ cells to facilitate immune responses $[2,3]$.

Macrophage-augmented inflammatory responses are associated with their differentiation and polarization. Monocytes differentiate to act both as phagocytes and APCs, and are involved in the clearance of pathogen-derived particles and toxins. Myeloid-derived monocytes migrate to different tissues through a C-C chemokine receptor 2initiated process, and then differentiate into unique macrophages $[4,5]$. Two primary macrophage subgroups have been proposed, termed classically activated macrophages (M1) and alternatively activated macrophages (M2). The M1 phenotype is characterized by IL- $12{ }^{\text {hi }} \mathrm{IL}-23^{\text {hi }} \mathrm{IL}-10^{\text {lo }}$ expression and iron uptake activity, facilitating inflammatory, microbicidal, and tumoricidal functions. M2 macrophages express IL-12 ${ }^{\text {lo }}$ IL-10 ${ }^{\text {hi }}$ IL-1decoyR ${ }^{\text {hi }}$ IL-1RA ${ }^{\text {hi }}$ and 
release iron, inhibiting inflammation, and promoting tissue remodeling and parasite clearance [6-8].

In chronic hepatitis $\mathrm{C}(\mathrm{CHC}), \mathrm{HCV}$ antigen stimulation leads to profound changes in host immune cells. For example, natural killer (NK) cells are directly inhibited by exposure to HCV E2 protein [9-11], while HCV core protein peptides facilitate HLA-E expression and thereby impair NK cell cytotoxicity $[12,13]$. HCV core and NS3 antigens activate monocytes to produce IL-10 and TNF- $\alpha$, as well as suppress IL-29 secretion, causing a functional inhibition of DCs $[14,15]$, and facilitating dimerization of Toll-like receptors (TLR) $1 / 2$ and $2 / 6$ and hepatic fibrogenesis [16-19]. Recent experiments indicated that HCV present in the sera of infected patients interferes with DC differentiation in vitro [20], indicating that such antigens may also interfere with macrophage function during differentiation.

Studies have shown that HCV antigens influence macrophage responses and cytokine expression, alter signaling receptors and adhesion molecule levels [21-23]. HCV also influences DC function by modulating their maturation and cytokine production [24]. Macrophage TLR-3, PD-1, and Tim-3 expression mediate HCV infection, leading to dysfunctional macrophage function $[25,26]$. However, whether $\mathrm{HCV}$ impacts macrophage polarization is still poorly understood.

$\mathrm{HCV}$ antigens affect macrophages through various signaling molecules. In this study we analyzed TNF- $\alpha$, IL-10, IL-12, transferrin receptor-1 (TfR1), and matrix metalloproteinases (MMPs) that are highly associated with macrophage polarization. We examined monocytes from healthy donors and $\mathrm{HCV}$ patients that were isolated and treated with IFN- $\gamma /$ lipopolysaccharide (LPS) to stimulate M1 polarization ex vivo. The data indicated that macrophages from $\mathrm{HCV}$ infected patients exhibited decreased TNF- $\alpha$ and TfR1 expression levels. HCV antigen stimulation of macrophages altered gene expression associated with M1 polarization, including the expression of TNF- $\alpha$, IL-10, IL-12, CD163, TfR1, MMPs and $\mathrm{CD} 100$, as well as inhibition of macrophage phagocytosis of $\mathrm{HCV}$ in cell culture (HCVcc). Furthermore, NF-kB activity was abnormally suppressed and A20 with its binding partner A20-binding inhibitor of NF- $\mathrm{KB}$ binding protein (ABIN1) was up regulated after polarization. A20/ABIN1 overexpression had a similar influence on $\mathrm{M} 1$ polarization compared with HCV antigen stimulation. As the A20/ ABIN1 complex negatively regulates NF- $\kappa B$ signaling [27, 28], HCV-induced A20/ABIN1 expression likely inhibits NF- $\kappa B$ and contributes to abnormal M1-polarization.

\section{Results}

Macrophages from HCV-infected patients are polarized abnormally ex vivo

Peripheral blood mononuclear cells (PBMC) from healthy donors $(n=29)$ were cultured with normal medium (HC group) or stimulation medium (HS group, containing $\mathrm{HCV}$-core protein and NS3/4A) for 1 day and were then treated with LPS/interferon (IFN) $-\gamma$ for M1 polarization. Macrophages were then isolated and analyzed by flow cytometry (Fig. 1a). As expected in response to LPS/IFN- $\gamma$ challenge, M1 polarization stimulated inflammatory cytokines (TNF- $\alpha$ and IL-12) [29-31], and TfR1 iron uptake receptors, and down-regulated scavenging receptors (MARCO, CD163, and CD23) [32-34]. However, compared with normal polarization, $\mathrm{HCV}$ antigen stimulation reduced TNF- $\alpha$ and TfR1 expression $(p<0.001)$, suggesting suppression of M1 polarization (Fig. 2b). Although CD163 and CD23 expression was not significantly different, a slight up-regulation was observed in some cases.

Macrophages from $\mathrm{CHC}$ patients receiving antiviral therapy (PT: $n=32$ ) were also tested. The patients were further divided into response and no response groups by alanine aminotransferase (ALT) levels (PTI group: ALT > 40U/L). Patient PBMCs were cultured with normal medium for 1 day prior to LPS/IFN- $\gamma$ M1 polarization. Macrophages isolated and analyzed by flow cytometry showed decreased TNF- $\alpha$ and TfR 1 levels (PT: TNF- $\alpha p<0.001$, PTI: TNF- $\alpha$ $p<0.001$ and PTI: TfR $1 p<0.05)$ (Fig. 1b), indicating impairment of M1-polarization. Nevertheless, CD163 and CD23 did not show much variation, which may indicate more complex cell-cell interactions in vivo.

\section{HCV antigens influence macrophage polarization by different mechanisms in vitro}

The impact of HCV antigens on M1 polarization was investigated using the THP-1 monocyte/macrophage system. TNF- $\alpha$, TfR1, CD163, CD23 and MMP expression levels were tested. The HCVcc and core protein showed different effects on TNF- $\alpha$ expression, as HCVcc enhanced TNF- $\alpha$ expression and the core protein reduced TNF- $\alpha$ expression (Fig. 2a). Nevertheless, the main effects of HCV antigens should be TNF- $\alpha$ inhibition according to ex vivo data that showed a decreasing trend in the patient group. An inhibitory effect was observed for TfR1 (Fig. 2b and Additional file 1: Figure S1). TfR1 mediates iron endocytosis, but decreased TfR1 expression resulting from $\mathrm{HCV}$ antigen stimulation blocks M1 iron uptake activity. CD163 and CD23 are important macrophage-specific receptors that switch between activated phenotypes during inflammation [35]. Although there was only a small change in CD163 and CD23 expression ex vivo, both were increased after incubation with $\mathrm{HCV}$ antigens (Fig. 2c, d) in vitro. M1 macrophages are characterized by high expression levels of TNF- $\alpha$ and TfR1, as well as low expression levels of CD163 and CD23. The variation of these molecules may have a long-term effect on the polarization process.

MMPs generally control extracellular matrix (ECM) remodeling and have extensive influence on macrophage 


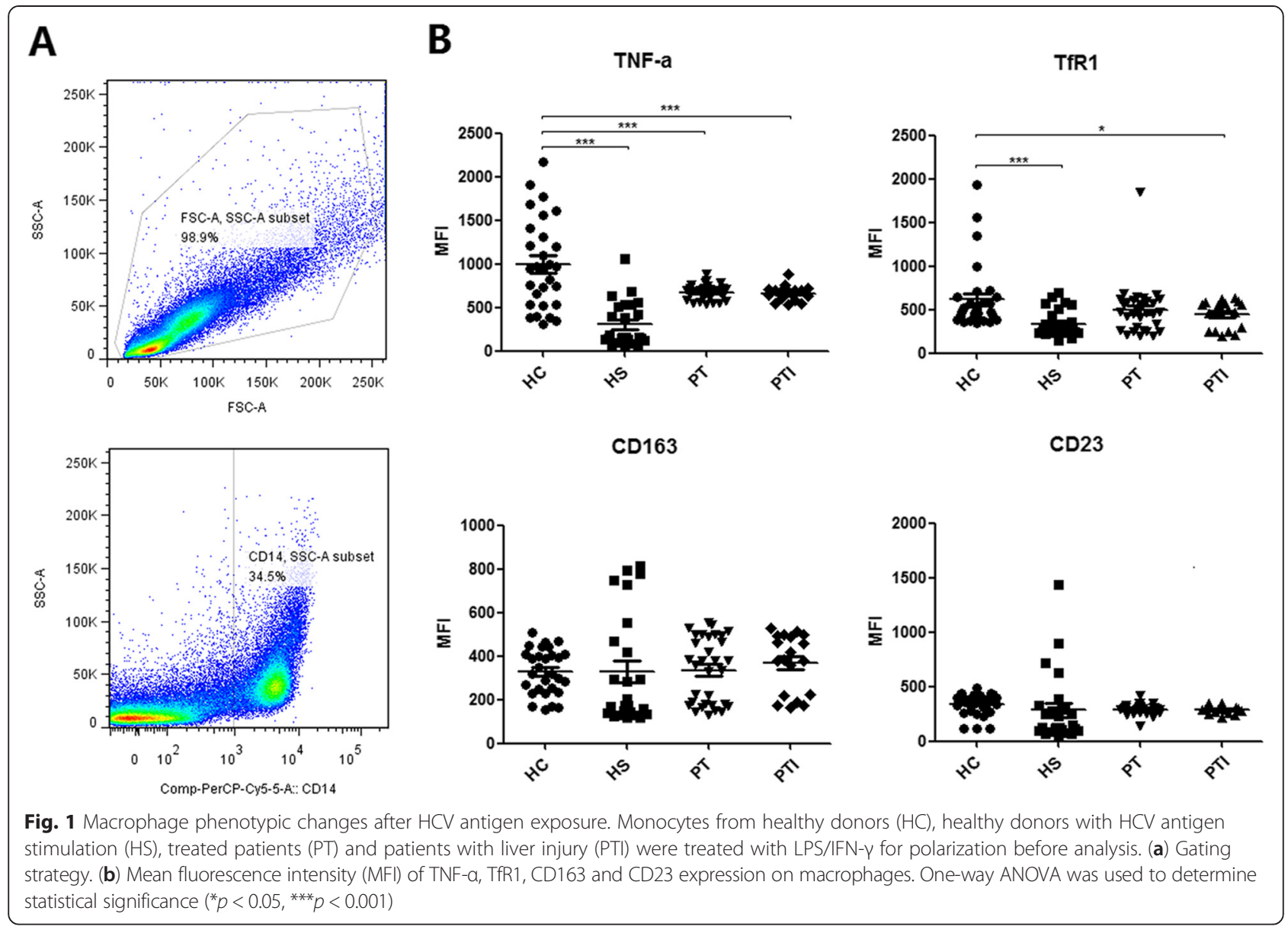

migration and cell metabolism [36]. Our data revealed that $\mathrm{HCV}$ core protein induced MMP2 expression, while HCVcc and NS3/4A had little effect. MMP3 expression decreased after core protein treatment and increased in NS3/4A-stimulated cells, while MMP7 expression inhibited $\mathrm{HCV}$ core protein and NS3/4A, and $\mathrm{HCVCC}$ stimulated its expression. Interestingly, MMP14 expression was elevated by all $\mathrm{HCV}$ antigens, which represents nonspecific changes in response to exogenous substances (Fig. 2e).

We next asked whether HCV stimulated expression of MMP14 target proteins. MMP14 digests CD100 from the macrophage cell surface to produce an essential source of soluble CD100 (sCD100) [37], which is a universal activator of immune cells. CD100 elicits T and B lymphocytes and NK cells by inhibiting the inhibitory receptor CD72, leading to the activation of target cells. SCD100 in particular detaches from the membrane and diffuses into the blood, and has greater inflammatory activity than its membrane form [38, 39]. However, although increased MMP14 expression increases SCD100 levels, total macrophage CD100 expression was restrained after HCV core and NS3/4A stimulation, suggesting extensive immune suppression by HCV antigens (Fig. 2f).
IL-10 and IL-12 expression levels were also altered in response to different $\mathrm{HCV}$ antigenic stimulation, indicating that abnormal polarization maybe induced by different antigens (Fig. 2g). Interestingly, IL-10 secretion was decreased following incubation with $\mathrm{HCV}$ antigens, implying a more significant M1-polarization (especially for the NS3/4A group), whereas HCV core stimulation reduced IL-12 secretion after polarization, suggesting impairment of M1-polarization. Macrophages are the primary source of IL-12 involved in Th1 and Th17 responses, and increased IL-12 expression is an important characteristic of M1 polarization [6, 40, 41]. Thus, a decrease in IL12 secretion caused by HCV core antigens likely dampens M1-induced inflammation.

HCV antigen pre-incubation influences A20/ABIN1 complex in $\mathrm{M} 1$ macrophages

Various signaling pathways influence macrophage maturation, including STATS [42-46], MAPK [47-49], and NF- $\kappa B$ [50, 51]. TLR engagement induces NF- $\kappa B$ activity, producing inflammatory mediators associated with $\mathrm{M} 1$ polarization. Conversely, M1 related gene suppression is commonly associated with blockage of NF-kB coactivators [52]. Our experiments tested NF- $\kappa B$ activity in 


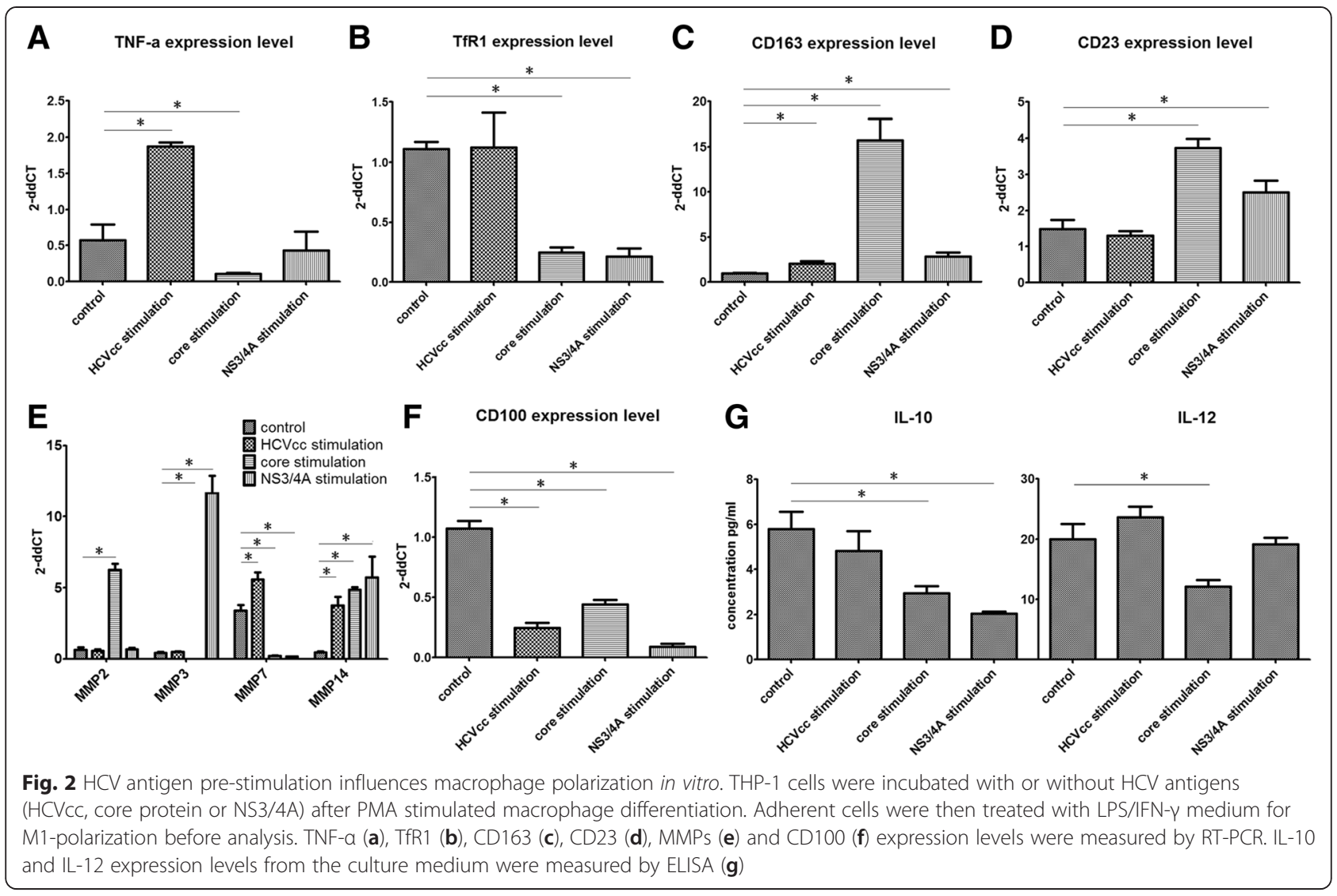

each group. Compared with normal M1 polarized cells, NF- $\kappa \mathrm{B}$ signaling occurred at a lower level (p65 phosphorylation) when pre-incubated with $\mathrm{HCV}$ antigens (Fig. 3b). We further checked expression of the A20/ ABIN1/TAX1BP1 complex, which regulates NF-кB activity. The data suggest that expression was distinctively influenced by each antigen (Fig. 3a). The HCV core protein significantly enhanced A20 expression, while $\mathrm{HCVcc}$ and NS3/4A contributed modestly. ABIN1 was up regulated by all antigens, while NS3/4A contributed more to efficiency. However, TAX1BP1 showed a slight reduction, especially when stimulated by NS3/4A. As the A20/ABIN1 complex suppresses NF- $\mathrm{kB}$ through the regulation of ubiquitin, which targets TBK1/IKKi, the total effect of $\mathrm{HCV}$ antigen stimulation should weaken NF-kB activity [28].

\section{Enhanced A20/ABIN1 contributes to abnormal M1 polarization}

We next determined whether overexpression of A20 and ABIN1 during macrophage differentiation induced similar effects to $\mathrm{HCV}$ antigen stimulation. A20/ABIN1 were overexpressed by adenovirus vectors in THP-1 cells and stimulated with phorbol myristate acetate (PMA). The expression efficiency was checked by co-expression marker green fluorescent protein (GFP) (Additional file 2: Figure S4). The overexpression of A20 and ABIN1 each inhibited NF- $\mathrm{kB}$ activity, as expected, whereas coexpression of the two proteins was more efficient. NF- $\mathrm{KB}$ activity gradually reduced following 3-day PMA stimulation (Fig. 4d). CD100, TfR1, IL-10, and IL-12 expression declined, while CD163 increased after A20/ABIN1 overexpression, correlating with $\mathrm{HCV}$ antigen stimulation (Fig. 4a, c). The influence on MMPs appears more complicated, as core MMP2 and MMP3 antigens, and MMP7 in the HCVcc group and MMP14 in all $\mathrm{HCV}$ antigens, showed a similar response to A20/ABIN1 overexpression, indicative of distinct regulatory mechanisms (Fig. 4b).

M2 inhibition of NF- $\mathrm{BB} / \mathrm{HIF}-1 \alpha$-dependent gene transcription is dependent upon STAT3/STAT6 activation [8]. During macrophage polarization, if A20/ABIN1 is overexpressed, NF- $\mathrm{kB}$ may be suppressed because of a negative A20/ABIN1 feedback loop, which may have profound influences on polarized macrophages. During $\mathrm{HCV}$ infection, $\mathrm{HCV}$ antigens exist in a number of organs as well as peripheral blood [53], suggesting that HCV stimulates A20/ABIN1-mediated inflammation via macrophages.

\section{A20/ABIN1 up-regulation dampens HCVcc phagocytosis of $\mathrm{M} 1$ macrophages}

As professional APCs, macrophages not only mediate nonspecific responses against microbes, but also induce 


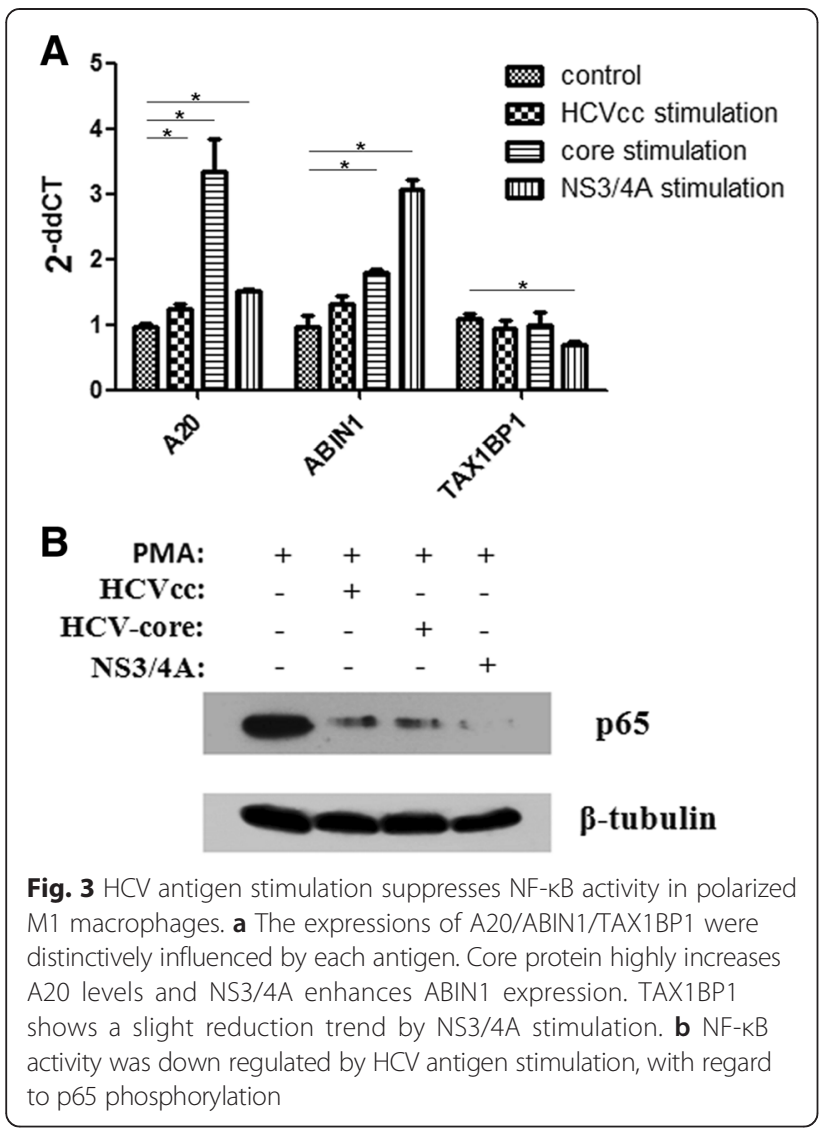

antigen-specific immune responses through antigen presentation. Macrophages present antigens that initiate B cell activation. Phagocytosis plays an essential role in this and is the first step of antigen presentation [54]. To examine whether premature macrophage- $\mathrm{HCV}$ antigen interaction impacts phagocytosis, stimulated THP-1 cells with or without $\mathrm{HCV}-\mathrm{NS} 3 / 4 \mathrm{~A}$ co-incubation were exposed to HCVcc for 6 hours. Cells were harvested and analyzed by flow cytometry. The results indicated that macrophages exposed to NS3/4A generated fewer HCVcc particles, suggesting reduced $\mathrm{HCV}$ phagocytosis (Fig. 5a and Additional file 3: Figure S2). Because HCV antigens stimulate A20/ ABIN1 expression, we further suppressed A20 by RNAi during stimulation and examined M1 phagocytosis activity by flow cytometry. Interestingly, an enhanced expression peak emerged, which suggests macrophage phagocytosis was rescued by A20 knockdown, indicating that A20 upregulation contributes to reduced phagocytosis (Fig. 5b and Additional file 4: Figure S3).

We next asked whether this reduction influenced viral concentrations in the ECM, which may be related to viral clearance. The culture medium viral titers were tested at each time point and after 72 hours co-incubation. HCVcc levels in antigen-exposed cells were significantly higher than normal controls $(p<0.001)$, suggesting that $\mathrm{HCV}$ clearance from ECM may be impaired (Fig. 5c). However, the extracellular medium viral titer did not change after A20 knockdown, suggesting that phagocytosis activity and ultimate ECM viral concentration may be determined by different mechanisms (Fig. 5d, upper).

\section{A20/ABIN1-mediated TfR1 reduction of macrophages is associated with extracellular HCVcc concentrations} TfR1 is ubiquitously expressed in most tissues, connecting the extracellular ironbound transferrin and endocytic adaptor TTP to facilitate clathrin-dependent endocytosis. Recent studies have shown that TfR1, besides its iron balance function, also participates in HCV entry of infected cells. As the A20/ABIN1 complex inhibits NF- $\mathrm{kB}$ signaling, which in turn regulates TfR1 expression through HIF-1 regulation, the change of HCV entry in M1 macrophages may be caused by a variation in TfR1 expression [55]. However, TfR1 overexpression does not rescue $\mathrm{HCVcc}$ phagocytosis from NS3/4A stimulated macrophages (Fig. 5d, lower). Although macrophage phagocytosis was rescued in A20 RNAi assays, the TfR1 expression level did not increase (Fig. 5e), suggesting that the macrophage phagocytosis process is influenced by A20/ABIN1 and is independent of a TfR 1 mediated process. Interestingly, although TfR 1 does not play a role in intracellular $\mathrm{HCV}$ endocytosis, it reduced the final extracellular concentration, as TfR1 overexpression decreased ECM viral titer, indicating that TfR1 likely mediates the active transport of HCVcc particles.

\section{Discussion}

Our study demonstrated that HCV-infected patients possess functionally defective M1 macrophages. Decreases in TNF- $\alpha$ and TfR 1 have positive effects on immune cell activation and iron uptake activity ex vivo, indicating suppression of the M1-inflammatory response. Furthermore, in vitro data indicated that $\mathrm{HCV}$ antigen exposure to monocytes influenced M1-polarization by decreasing IL-12, CD100 and TfR1, as well as up-regulating CD163 and MMPs, implying compromised cell polarization. Thus, HCV antigen stimulation seems to inhibit inflammatory cytokines and iron uptake activity, facilitate antiinflammatory receptor expression; taken together this characterizes an M1-polarization antagonized phenotype. Notably, rather than inducing macrophage transformation from M1 to M2, HCV stimulation caused a dysfunction of the inflammatory process which both impair M1 and M2 polarization, regarding to weakened phagocytosis activity (Fig. 5) which would be enhanced in transformation of M1 to M2 [6-8].

The liver contains large numbers of tissue-resident macrophages, Kupffer cells, which might be impacted similarly. Kupffer cells contribute to immune regulation in the liver. They express immune sensing receptors, through which they contribute to inflammatory induction in the 


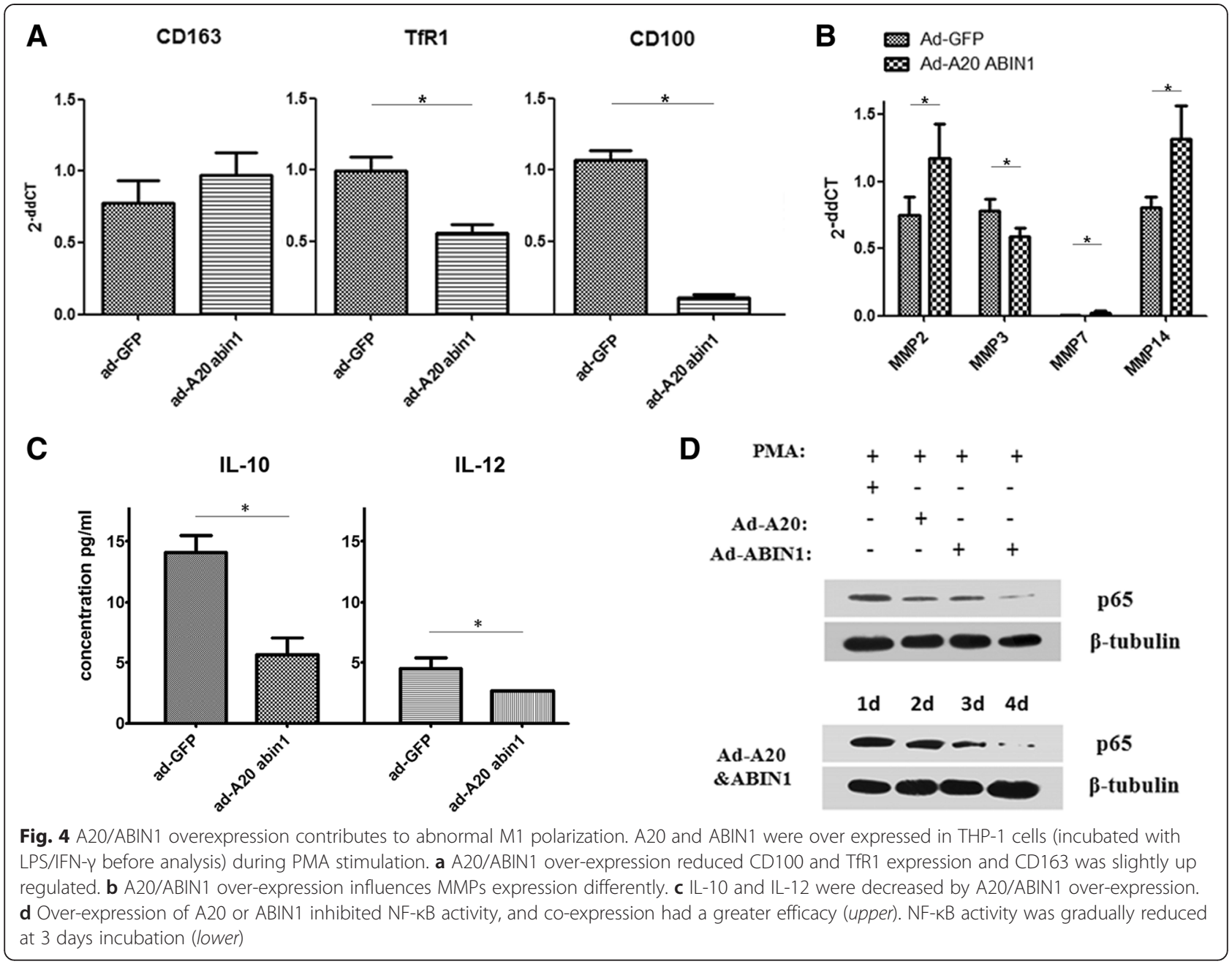

liver by releasing pro-inflammatory mediators [56]. At the same time, they also express potent anti-inflammatory cytokines and contribute to the local regulation of innate and adaptive immune responses [57-60]. HCV antigen stimulation induces anti-inflammatory processes that contribute to chronic HCV infection in the liver.

$\mathrm{HCV}$-infected patients often exhibit insufficient immune responses in the clinic. During the early stages of infection, circulating $\mathrm{HCV}$ RNA is detected 3-7 days after exposure, but seroconversion occurs weeks or months after infection, implying HCV immune suppressive effects. Indeed, humans possess a negative feedback loop in response to inflammation. However, in HCV infection, this negative response may be too sensitive to stimulate an efficient inflammatory response, which requires extra drugs such as PEG-IFN- $\alpha$. According to our data, the exposure of HCV antigens on monocytes probably contributes to anti-inflammatory processes during $\mathrm{HCV}$ infection.

To further interpret the mechanism of $\mathrm{HCV}$ antigen induced macrophage variation, NF- $\mathrm{kB}$ activity was analyzed. NF- $\mathrm{kB}$ signaling plays an essential role in immune regulation and macrophage polarization [55]. Following HCV antigen stimulation, M1-polarized macrophages exhibited an A20/ABIN1 up-regulated phenotype, which suppresses NF- $\mathrm{kB}$ activity. Antigen stimulation was antigen-specific, as $\mathrm{HCV}$ core protein increased A20, whereas NS3/4A up-regulatedABIN1. As expected, overexpression of A20/ABIN1 generated a similar phenotype to that by $\mathrm{HCV}$ antigen stimulation, including decreased CD100, TfR1, IL-10 and IL-12 expression, and increased CD163 and MMP14 expression, as well as reduced phagocytosis. Summarily, A20/ABIN1 up-regulation induced by $\mathrm{HCV}$ antigens suppressed NF- $\mathrm{kB}$ activity and influenced macrophage differentiation.

Interestingly, A20 knockdown rescued phagocytosis, but had less effect on medium virus concentrations, whereas TfR1 overexpression reduced extracellular viral load. The iron regulator TfR 1 is an important HCV entry factor [55]; thus, TfR1 may be involved in macrophagemediated HCV phagocytosis, or may impact HCV clearance via the ECM. 


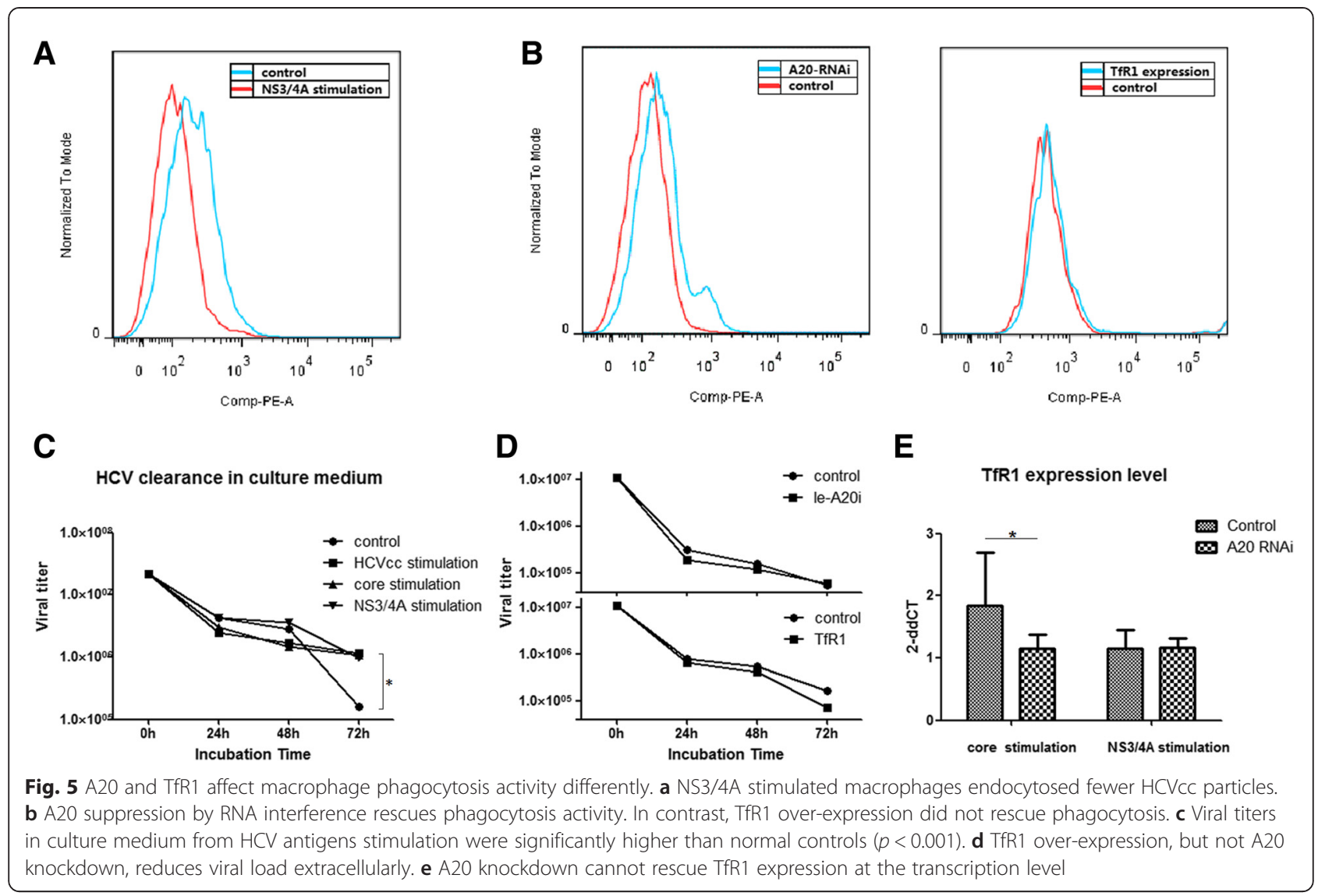

\section{Materials and methods \\ Subjects}

Study subject information is summarized in Additional file 5: Table S1. The first group comprised 30 female healthy donors, the second group included $7 \mathrm{HCV}$ infected patients who were untreated (treatment-naive patients), and the third group included 32 patients who had been undergoing IFN- $\alpha$ /ribavarin antiviral therapy for 1-6 months (patients receiving antiviral treatment, PT). All patients and healthy donors were negative for HIV and hepatitis B virus. Testing of ALT and HCV viral load was performed by the Department of Infectious Diseases at Tangdu Hospital. The protocol conformed to the ethical guidelines of 1975 Declaration of Helsinki and was approved by the Research and Ethical Committee of Tangdu Hospital of the Fourth Military Medical University. Informed consent was obtained from all donors.

\section{Cells and stimulation}

PBMCs were isolated from blood with Ficoll-Hypaque density centrifugation (Sigma, St Louis, MO, USA) according to the manufacturer's protocol. Healthy donors PBMCs were cultured with X-vivo 15 medium (Lonza, Walkersville, MD, USA) or stimulation medium (X-vivo 15 containing $\mathrm{HCV}$-core protein and NS3/4A,
American Research Products Inc. Waltham, MA, USA) for 1 day and were then treated with LPS/IFN- $\gamma$ for M1 polarization. Patient PBMCs were cultured with X-vivo 15 medium for 1 day prior to LPS/IFN- $\gamma$ M1 polarization.

THP-1 cells cultured in RPMI 1640 (Mediatech Inc, Manassas, VA, USA) were obtained from ATCC (Manassas, VA, USA). Huh7.5 human hepatoma cells (ATCC) were grown in Dulbecco's Modified Eagle's Medium (Mediatech Inc.) containing $10 \%$ fetal bovine serum (Life Technologies, Grand Island, NY, USA), $100 \mu \mathrm{g} / \mathrm{ml}$ penicillin-streptomycin, $0.05 \mathrm{mM} \quad \beta$-mercaptoethanol and $2 \mathrm{mM} \mathrm{L}$-glutamine (Mediatech Inc.). Cells were cultured at $37{ }^{\circ} \mathrm{C}$ with $5 \% \mathrm{CO}_{2}$. For cell stimulation, THP1 cells were cultured for 3 days in 1640 medium with PMA for differentiation $[61,62]$ with or without HCV-core, NS3/4A (American Research Products Inc. Waltham, MA, USA) or HCVcc. Cells were then cultured in RPMI 1640 containing IFN- $\gamma /$ LPS for M1 polarization [63-65] 1 day before analysis.

\section{HCVcc generation and vector construction}

HCVcc was generated by transfection of the HCV pJFH1 transcript (generated by TranscriptAid T7 High Yield Transcription kit, ThermoFisher Scientific, Waltham, MA, US) into Hu7.5 cells using DMRIE-C reagents 
(Invitrogen, Carlsbad, CA, USA), A20 and ABIN1 adenovirus (ad-A20 and ad-ABIN1), A20 RNAi lentiviral (le-A20i), and TfR1 (pGV146-tfr1) overexpression vectors were purchased from Genechem Co. Ltd (Shanghai, China).

\section{Western blotting}

Cells were harvested in lysis buffer $(50 \mathrm{mM}$ Tris- $\mathrm{HCl}$ at $\mathrm{pH}$ 7.5, $150 \mathrm{mM} \mathrm{NaCl}, 2 \mathrm{mM}$ EDTA) supplemented with protease inhibitor (Roche Applied Science, Indianapolis, IN, USA). Lysate was run by $10 \%$ sodium dodecyl sulfate polyacrylamide gel electrophoresis and transferred to polyvinylidene difluoride membranes (Immobilon-P, Millipore, Billerica, MA, USA). Membranes were blocked with $5 \%$ (wt/vol) non-fat milk followed by incubation with primary antibodies (p-NF-kB p65 mouse mAb, Cell Signaling, Danvers, MA, USA) and secondary antibody (horseradish peroxidase-conjugated goat anti-mouse, Abgent, San Diego, CA, USA). Membranes probed with $\beta$-actin monoclonal antibody (Bioworld Technology, St. Louis Park, MN, USA) served as a control.

\section{Immunofluorescence}

Cells in chamber slide wells were fixed with paraformaldehyde and stained as follows. Primary antibodies against TfR1 (eBioscience, San Diego, CA, USA) and HCV core (mAb to HCV core Antigen, Abcam, Cambridge, MA, USA) were incubated at a 1:2000 dilution overnight at $4{ }^{\circ} \mathrm{C}$. Conjugated secondary antibodies (anti-mouse Alexa-555, Abcam) were incubated at a 1:1000 dilution for $1 \mathrm{~h}$ at room temperature. Nuclei were stained with 4',6diamidino-2-phenylindole (DAPI). Images were captured via confocal microscopy (BX51-DP70 system, Olympus, Tokyo, Japan) and analyzed using Olympus DP Controller and Management software.

\section{RNA isolation and RT-PCR Analysis}

RNA was purified using RNeasy Mini Kit (Qiagen, Gaithersburg, MD, USA) according to the manufacturer's instructions. After DNase I (Invitrogen) treatment, cDNA was synthesized by RevertAid First Strand cDNA Synthesis Kit (ThermoFisher Scientific) following the manufacturer's protocol. Gene expression was measured by RT-qPCR with SYBR Premix Ex Taq II (Takara, Mountain View, CA, USA), using Bio-Rad iQ5 Real-time Thermocycler as described (Bio-Rad, Hercules, CA, USA). Expression levels were determined relative to a standard curve and normalized to GAPDH. Primer sequences are shown in Additional file 6: Table S2.

\section{Flow cytometry}

Cells were fixed with $2 \%$ paraformaldehyde and permeable reagent (eBioscience) for $30 \mathrm{~min}$ on ice. Cells were stained with fluorescently labeled antibodies $(1 \mu \mathrm{g} / \mathrm{ml}$;
$1 \mathrm{~h}$ at room temperature), assayed by flow cytometry (FASCAria II, BD Bioscience, Franklin Lakes, NJ, USA), and data were analyzed by Flowjo7.6 software (Tree Star, Inc. Ashland, OR, USA). Primary antibodies used for staining were anti-human cd71-FITC (eBioscience), mAb to $\mathrm{HCV}$ core Antigen (Abcam), and goat anti-mouse Alexa555 antibody (Abcam).

\section{ELISA}

IL-10 and IL-12 concentrations in stimulated cell culture medium were determined by human IL-10 or IL-12 Platinum ELISA kit (eBioscience). Duplicate standard curves were prepared as follows. Briefly, diluted standard proteins were added to plates, incubated at $4{ }^{\circ} \mathrm{C}$ overnight, blocked, and antibodies were added. After addition of substrate solution, reactions were stopped by adding $25 \mu \mathrm{l} 2 \mathrm{M} \mathrm{H}_{2} \mathrm{SO}_{4}$. Results were detected by micro-plate reader (BioTek; Shanghai, China) and IL-10 and IL-12 concentrations were calculated against the standard curve by CurveExpert1.3 [19].

\section{Statistical analysis}

One-way ANOVA and Student's $t$-test were used to determine statistical significance. Analyses were performed with GraphPad Prism Version 5.0 (La Jolla, CA, USA) and SPSS Statistics 21.0 (IBM Corp., Armonk, NY, USA). A $p$ value $<0.05$ was considered statistically significant.

\section{Conclusions}

In summary, this study demonstrated that $\mathrm{HCV}$ antigens impact M1 macrophage polarization. After antigen stimulation, NF-kB signaling is suppressed by upregulation of the A20/ABIN1 complex, which leads to variation of functional molecules such as CD163, MMPs, TfR1, and CD100, indicating an anti-inflammatory reaction against M1-polarization.

\section{Additional files}

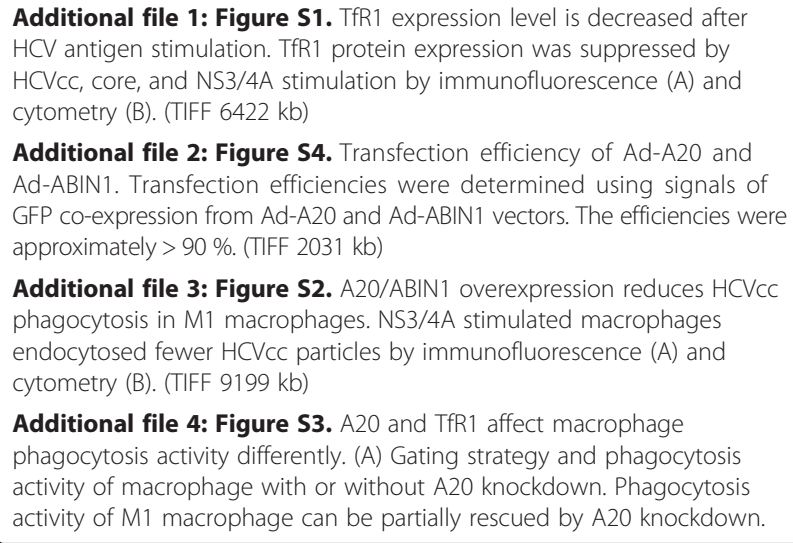

Additional file 1: Figure S1. TfR1 expression level is decreased after HCV antigen stimulation. TfR1 protein expression was suppressed by HCVcC, core, and NS3/4A stimulation by immunofluorescence (A) and cytometry (B). (TIFF 6422 kb)

Additional file 2: Figure S4. Transfection efficiency of Ad-A20 and Ad-ABIN1. Transfection efficiencies were determined using signals of GFP co-expression from Ad-A20 and Ad-ABIN1 vectors. The efficiencies were approximately $>90 \%$. (TIFF $2031 \mathrm{~kb}$ )

Additional file 3: Figure S2. A20/ABIN1 overexpression reduces HCVCC phagocytosis in M1 macrophages. NS3/4A stimulated macrophages endocytosed fewer HCVcc particles by immunofluorescence (A) and cytometry (B). (TIFF 9199 kb)

Additional file 4: Figure S3. A20 and TfR1 affect macrophage phagocytosis activity differently. (A) Gating strategy and phagocytosis activity of macrophage with or without A20 knockdown. Phagocytosis activity of M1 macrophage can be partially rescued by A20 knockdown. 
(B) Gating strategy and phagocytosis activity of macrophage with or without TfR1 overexpression. No difference was detected in TfR1 overexpressing cells. (Positive cells with TfR1 overexpression vector were selected by FITC marker first; phagocytosis activities are indicated by anti-HCV core in PE channel). (TIFF $16175 \mathrm{~kb}$ )

Additional file 5: Table S1. Characteristics of the study subjects. (XLS $18 \mathrm{~kb}$ )

Additional file 6: Table S2 Primers used in the experiment. (XLS 20 kb)

\section{Competing interests}

The authors declare that they have no competing interests.

\section{Authors' contributions}

$\mathrm{ZJ}$ and CF participated in the design and conducted the majority of the experiments and drafted the manuscript. Y Zhang contributed to the interpretation of the findings and revised the manuscript. Y Zhou, BL, YH and YG performed analyses of data. All authors read and approved the final manuscript.

\section{Acknowledgements}

The authors acknowledge the financial support provided by National Natural Science Foundation of China $(81170389,81300314,81270499)$. The authors thank Dr. Charles M. Rice for kindly providing reagents.

Received: 11 June 2015 Accepted: 1 September 2015 Published online: 17 September 2015

\section{References}

1. Shepard CW, Finelli L, Alter MJ. Global epidemiology of hepatitis C virus infection. Lancet Infect Dis. 2005;5:558-67.

2. Kapsenberg ML. Dendritic-cell control of pathogen-driven T-cell polarization. Nat Rev Immunol. 2003;3:984-93.

3. Batista FD, Harwood NE. The who, how and where of antigen presentation to B cells. Nat Rev Immunol. 2009;9:15-27.

4. Serbina NV, Pamer EG. Monocyte emigration from bone marrow during bacterial infection requires signals mediated by chemokine receptor CCR2. Nat Immunol. 2006;7:311-7.

5. Ancuta P, Liu KY, Misra V, Wacleche VS, Gosselin A, Zhou X, et al. Transcriptional profiling reveals developmental relationship and distinct biological functions of CD16+ and CD16- monocyte subsets. BMC Genomics. 2009;10:403.

6. Subhra KB, Manesh C, Irina NS, Lim JY. Macrophage polarization and plasticity in health and disease. Immunol Res. 2012;53:11-24.

7. Gordon S, Martinez FO. Alternative activation of macrophages: mechanism and functions. Immunity. 2010;32:593-604

8. Sica A, Mantovani A. Macrophage plasticity and polarization in vivo. J Clin Invest. 2012;122:787-95.

9. Crotta S, Stilla A, Wack A, D'Andrea A, Nuti S, D'Oro U, et al. Inhibition of natural killer cells through engagement of $C D 81$ by the major hepatitis $C$ virus envelope protein. J Exp Med. 2002;195:35-41.

10. Tseng $C T$, Klimpel GR. Binding of the hepatitis $C$ virus envelope protein $E 2$ to CD81 inhibits natural killer cell functions. J Exp Med. 2002;195:43-9.

11. Krämer B, Schulte D, Körner C, Zwank C, Hartmann A, Michalk M, et al. Regulation of NK cell trafficking by CD81. Eur J Immunol. 2009;39:3447-58.

12. Nattermann J, Nischalke HD, Hofmeister V, Ahlenstiel G, Zimmerman $H$, Leifeld $L$, et al. The HLA-A2 restricted T cell epitope HCV core 35-44 stabilizes HLA-E expression and inhibits cytolysis mediated by natural killer cells. Am J Pathol. 2005;166:443-53.

13. Nattermann J, Feldmann G, Ahlenstiel G, Langhans B, Sauerbruch T, Spengler U. Surface expression and cytolytic function of natural killer cell receptors is altered in chronic hepatitis C. Gut. 2006;55:869-77.

14. Langhans B, Kupfer B, Braunschweiger I, Arndt S, Schulte W, Nischalke $H D$, et al. Interferon-lambda serum levels in hepatitis C. J Hepatol. 2011;54:859-65.

15. Dolganiuc A, Chang S, Kodys K, Mandrekar P, Bakis G, Cormier M, et al. Hepatitis C virus (HCV) core protein-induced, monocyte-mediated mechanisms of reduced IFN-alpha and plasmacytoid dendritic cell loss in chronic HCV infection. J Immunol. 2006;177:6758-68.
16. Chang S, Dolganiuc A, Szabo G. Toll-like receptors 1 and 6 are involved in TLR2-mediated macrophage activation by hepatitis C virus core and NS3 proteins. J Leukoc Biol. 2007;82:479-87.

17. Varchetta S, Mele D, Mantovani S, Oliviero B, Cremonesi E, Ludovisi S, et al. Impaired intrahepatic natural killer cell cytotoxic function in chronic hepatitis C virus infection. Hepatology. 2012;56:841-9.

18. Ma CJ, Lei N, Zhang Y, Zhang CL, Wu XY, Atia AN, et al. PD-1 negatively regulates interleukin-12 expression by limiting STAT-1 phosphorylation in monocytes/macrophages duringchronic hepatitis $C$ virus infection. Immunology. 2011;132:421-31.

19. Zhang Y, Ma CJ, Lei N, Zhang CL, Wu XY, Zhang Y, et al. Cross-talk between programmed death-1 and suppressor of cytokine signaling-1 in inhibition of il-12 production by monocytes/macrophages in hepatitis c virus infection. J Immunol. 2011;186:3093-103.

20. Granato M, Lacconi V, Peddis M, Renzo LD, Valia S, Rivanera D, et al. Hepatitis $C$ virus present in the sera of infected patients interferes with the autophagic process of monocytes impairing their in-vitro differentiation into dendritic cells. Biochim Biophys Acta. 1843;2014:1348-55.

21. Preisser L, Miot C, Guillou HL, Beaumont E, Foucher ED, Garo E, et al. IL-34 and macrophage colony-stimulating factor are overexpressed in hepatitis $C$ virus fibrosis and induce profibrotic macrophages that promote collagen synthesis by hepatic stellate cells. Hepatology. 2014;60:1879-90.

22. Negash AA, Ramos HJ, Crochet N, Lau DT, Doehle B, Papic N, et al. IL-1B production through the NLRP3 inflammasome by hepatic macrophages links hepatitis $C$ virus infection with liver inflammation and disease. PLoS Pathog. 2013;9, e1003330.

23. Shrivastava $S$, Mukherjee A, Ray R, Raya RB. Hepatitis $C$ virus induces interleukin-1 $(\mathrm{IL}-1 \beta) / \mathrm{LL}-18$ in circulatory and resident liver macrophages. J Virol. 2013;87:12284-90.

24. Ma L, Zhou Y, Zhang Y, Jia ZS. Role of A20 in IFN-a-mediated functional restoration of myeloid dendritic cells in patients with chronic hepatitis $C$. Immunology. 2014;143:670-8.

25. Qian F, Bolen CR, Jing CX, Wang XM, Wei Z, Zhao HY, et al. Impaired toll-like receptor 3-mediated immune responses from macrophages of patients chronically infected with hepatitis $\mathrm{c}$ virus. Clin Vaccine Immunol. 2013;20:146-55.

26. Zhang Y, Ma CJ, Wang JM, Ji XJ, Wu XY, Jia ZS, et al. Tim-3 negatively regulates IL-12 expression by monocytes in HCV infection. PLoS One. 2011;6:e19664.

27. Igarashi H, Yahagi A, Saika T, Hashimoto J, Tomitab T, Yoshikaw H, et al. A pro-inflammatory role for A20 and ABIN family proteins inhuman fibroblastlike synoviocytes in rheumatoid arthritis. Immunol Lett. 2011;141:246-53.

28. Gao L, Coope H, Grant S, Ma A, Ley SC, Harhaj EW. ABIN1 protein cooperates with TAX1BP1 and A20 proteins to inhibit antiviral signaling. J Biol Chem. 2011;286:36592-602.

29. Fresno C, Garcia RF, Gomez PV, Soares SA, Fernandez RI, Jurado T, et al. Potent phagocytic activity with impaired antigen presentation identifying lipopolysaccharide-tolerant human monocytes: demonstration in isolated monocytes from cystic fibrosis patients. J Immunol. 2009;182:6494-507.

30. Porta C, Rimoldi M, Raes G, Brys L, Ghezzi P, Liberto D, et al. Tolerance and M2 (alternative) macrophage polarization are related processes orchestrated by p50 nuclear factor kappaB. Proc Natl Acad Sci U S A. 2009;106:4978-83.

31. Pena OM, Pistolic J, Raj D, Fjell CD, Hancock RE. Endotoxin tolerance represents a distinctive state of alternative polarization (M2) in human mononuclear cells. J Immunol. 2011;186:7243-54.

32. Biswas SK, Lopez-Collazo E. Endotoxin tolerance: new mechanisms, molecules and clinical significance. Trends Immunol. 2009;30:475-87.

33. Cavaillon JM, Adib-Conquy M. Bench-to-bedside review: endotoxin tolerance as a model of leukocyte reprogramming in sepsis. Crit Care. 2006;10:233. London, England.

34. Biswas SK, Chittezhath M, Shalova IN, Lim JY. Macrophage polarization and plasticity in health and disease. Immunol Res. 2012;53:11-24.

35. Etzerodt A, Moestrup SK. CD163 and inflammation: biological, diagnostic, and therapeutic aspects. Antioxidants\&Redox Signaling. 2013;18:352-63.

36. Gurtner GC, Werner S, Barrandon Y, Longaker MT. Wound repair and regeneration. Nature. 2008:453:314-21.

37. Basile JR, Holmbeck K, Bugge TH, Gutkind JS. MT1-MMP controls tumorinduced angiogenesis through the release of semaphorin 4D. J Biol Chem. 2007;282:6899-905.

38. Kumanogoh A, Kikutani $\mathrm{H}$. The CD100-CD72 interaction: a novel mechanism of immune regulation. Trends Immunol. 2001;22:670-6. 
39. Witherden DA, Watanabe M, Garijo O, Rieder SE, Sarkisyan G, Cronin SJ, et al. The CD100 receptor interacts with its plexin B2 ligand to regulate epidermal $\gamma \delta$ T cell function. Immunity. 2012;37:314-25.

40. Krausgruber T, Blazek K, Smallie T, Alzabin S, Lockstone H, Sahgal N, et al. IRF5 promotes inflammatory macrophage polarization and TH1-TH17 responses. Nat Immunol. 2011;12:231-8.

41. Auffray C, Fogg D, Garfa M, Elain G, Join-Lambert O, Kayal S, et al. Monitoring of blood vessels and tissues by a population of monocytes with patrolling behavior. Science. 2007;317:666-70.

42. Xu H, Zhu J, Smith S, Foldi J, Zhao B, Chung AY, et al. Notch-RBP-J signaling regulates the transcription factor IRF8 to promote inflammatory macrophage polarization. Nat Immunol. 2012;13:642-50.

43. Kozicky LK, Sly LM. Phosphatase regulation of macrophage activation. Semin Immunol. 2015; doi:10.1016/j.smim.2015.07.001.

44. Satoh T, Takeuchi O, Vandenbon A, Yasuda K, Tanaka Y, Kumagai Y, et al. The Jmjd3-Irf4 axis regulates M2 macrophage polarization and host responses against helminth infection. Nat Immunol. 2010;11:936-44.

45. Serezani CH, Lewis C, Jancar S, Peters-Golden M. Leukotriene B4 amplifies NF-kappaB activation in mouse macrophages by reducing SOCS1 inhibition of MyD88 expression. J Clin Invest. 2011;121:671-82.

46. Spence S, Fitzsimons A, Boyd CR, Kessler J, Fitzgerald D, Elliott J, et al. Suppressors of cytokine signaling 2 and 3 diametrically control macrophage polarization. Immunity. 2013;38:66-78.

47. Zhang W, Xu W, Xiong S. Blockade of Notch1 signaling alleviates murine lupus via blunting macrophage activation and M2b polarization. J Immunol. 2010;184:6465-78.

48. Zhang W, Xu W, Xiong S. Macrophage differentiation and polarization via phosphatidylinositol 3-kinase/Akt-ERK signaling pathway conferred by serum amyloid P component. J Immunol. 2011;187:1764-77.

49. Wang XN, Pesakhov S, Harrisonc JS, Kafk M, Danilenko M, Studzinski GP. The MAPKERK5, but not ERK1/2, inhibits the progression of monocytic phenotype to the functioning macrophage. Exp Cell Res. 2015;30:199-211.

50. Smale ST. Selective transcription in response to an inflammatory stimulus. Cell. 2010;140:833-44.

51. Zhang W, Liu H, Liu W, Liu Y, Xu J. Polycomb-mediated loss of microRNA let-7c determines inflammatory macrophage polarization via PAK1dependent NF-jB pathway. Cell Death Differ. 2015;22:287-97.

52. Mahabeleshwar GH, Kawanami D, Sharma N, Takami Y, Zhou G, Shi H, et al. The myeloid transcription factor KLF2 regulates the host response to polymicrobial infection and endotoxic shock. Immunity. 2011:34:715-28.

53. Attallah AM, Abdallah SO, El-Far M, Omran MM, Tabll AA, Ghaly MF, et al. Perinatal transmission of hepatitis $C$ antigens: envelope 1, envelope 2 and non-structural 4. Infect Dis (Lond). 2015;29:1-7.

54. Xu W, Banchereau J. The antigen presenting cells instruct plasma cell Differentiation. Front Immunol. 2014;4:504.

55. Martin DN, Uprichard SL. Identification of transferrin receptor 1 as a hepatitis C virus entry factor. Proc Natl Acad Sci U S A. 2013;110:10777-82.

56. Petrasek J, Bala S, Csak T, Lippai D, Kodys K, Menashy V, et al. IL-1 receptor antagonist ameliorates inflammasome-dependent alcoholic steatohepatitis in mice. J Clin Invest. 2012;122:3476-89.

57. Knolle PA, Uhrig A, Protzer U, Trippler M, Duchmann R, Meyer zum Buschenfelde $\mathrm{KH}$, et al. Interleukin-10 expression is autoregulated at the transcriptional level in human and murine Kupffer cells. Hepatology. 1998;27:93-9.

58. You Q, Cheng L, Kedl RM, Ju C. Mechanism of T cell tolerance induction by murine hepatic kupffer cells. Hepatology. 2008;48:978-90.

59. Breous E, Somanathan S, Vandenberghe LH, Wilson JM. Hepatic regulatory $T$ cells and Kupffer cells are crucial mediators of systemic T cell tolerance to antigens targeting murine liver. Hepatology. 2009;50:612-21.

60. Wiegard C, Frenzel C, Herkel J, Kallen KJ, Schmitt E, Lohse AW. Murine liver antigen presenting cells control suppressor activity of CD4 + CD25 + regulatory T cells. Hepatology. 2005;42:193-9.

61. Daigneault M, Preston JA, Marriott HM, Whyte MB, Dockrell DH. The identification of markers of macrophage differentiation in PMAstimulated THP-1 cells and monocyte-derived macrophages. PLoS One. 2010;5:e8668.

62. Stokes RW, Doxsee D. The receptor-mediated uptake, survival, replication, and drug sensitivity of Mycobacterium tuberculosis within the macrophagelike cell line THP-1: a comparison with human monocyte-derived macrophages. Cell Immunol. 1999;197:1-9.
63. Vuletic S, Dong W, Wolfbauer G, Tang C, Albers JJ. PLTP regulates STAT3 and NF-KB in differentiated THP1 cells and human monocyte-derived macrophages. Biochim Biophys Acta. 1813;2011:1917-24.

64. Rodriguez-Prados JC, Traves PG, Cuenca J, Rico D, Aragones J, Martin-Sanz P, et al. Substrate fate in activated macrophages: a comparison between innate, classic, and alternative activation. J Immunol. 2010;185:605-14.

65. Cairo G, Recalcati S, Mantovani A, Locati M. Iron trafficking and metabolism in macrophages: contribution to the polarized phenotype. Trends Immunol. 2011;32:241-7.

\section{Submit your next manuscript to BioMed Central and take full advantage of:}

- Convenient online submission

- Thorough peer review

- No space constraints or color figure charges

- Immediate publication on acceptance

- Inclusion in PubMed, CAS, Scopus and Google Scholar

- Research which is freely available for redistribution 